\title{
Assessment of the Effectiveness of Ergonomics Training on the Improve- ment of Work Methods Among Hospital Office Staff
}

\author{
Mahshid Bahrami ${ }^{1}$, Mansoureh Sadeghi ${ }^{1}$, Alireza Dehdashti ${ }^{*}$, Meysam Karami ${ }^{3}$
}

1. BSc Studentm, Department of Occupational Health Engineering, Faculty of Health, Semnan University of Medical Sciences, Semnan, Iran

2. Associate Professor, Social Determinants of Health Research Center, Semnan University of Medical Sciences, Semnan, Iran

3. BSc Student, Department of Environmental Health Engineering, Faculty of Health, Semnan University of Medical Sciences, Semnan, Iran

\begin{tabular}{ll}
\hline \multicolumn{2}{c}{ Article Info } \\
\hline Original Article \\
Received: & $2018 / 07 / 13$ \\
Accepted: & $2018 / 09 / 23$ \\
Published Online: & $2018 / 09 / 23$
\end{tabular}

DOI: $10.30699 /$ jegon.6.2.34

Use your device to scan and read the article online

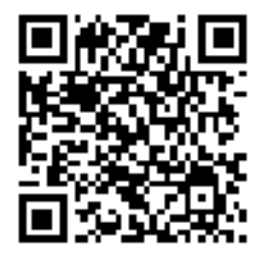

Corresponding Information

\section{Alireza Dehdashti}

Associate Professor, Social Determinants of Health Research Center, Semnan University of Medical Sciences, Semnan, Iran

Email:

dehdashti@semums.ac.ir

\begin{abstract}
Introduction: working with computer for long duration, static condition, and poor posture may lead to musculoskeletal disorders. The aim of this study was to assess an educational and ergonomic intervention on improving work procedure among computer users in office work in 2016.
\end{abstract}

Materials \& Methods: This descriptive-analytic study was carried out on 28 employees of administrative staff of Damghan Velayat Hospital. In order to determine the level of ergonomic risk, the physical status of patients was evaluated before and 6 months after intervention using ROSA method. The ergonomic principles were also used to increase the staff's awareness. Finally, one-way analysis of variance and independent t-test were used to analyze the statistical data.

Results: Prior to the implementation of the educational intervention program, the most important risk factors were in the neck $(P=0.009)$, elbows $(P=0.019)$ and shoulders $(P=0.031)$, respectively. After the intervention, this level of risk was reduced in the organs, which was a significant change. There were also other variables; age $(P=0.028)$, gender $(P=0.015)$, BMI $(P=0.040)$, education level $(P=0.047)$ and job experience $(P=0.025)$. They had a significant effect on the final score of ROSA.

Conclusion: The results indicated the prevalence of musculoskeletal disorders and ergonomic risk level by ROSA method which requires ergonomic intervention in the design of the environment, tools and equipment along with educational intervention.

Keywords: Ergonomic, Educational Intervention, Administrative staff, ROSA assessment method

Copyright (C) 2018, Journal of Ergonomics. This is an open-access article distributed under the terms of the Creative Commons Attribution-noncommercial 4.0 International License which permits copy and redistribute the material just in noncommercial usages, provided the original work is properly cited

How to Cite This Article:

Bahrami M, Sadeghi M, Dehdashti A, Karami M. Assessment of the Effectiveness of Ergonomics Training on the Improvement of Work Methods Among Hospital Office Staff. J Ergon. 2018; 6 (2): 34-45 


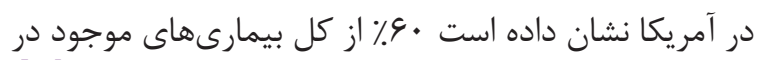

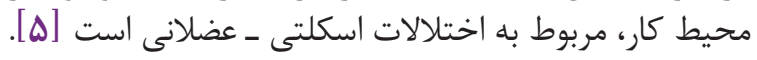

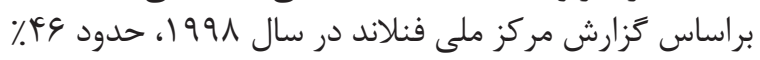

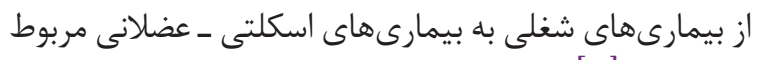

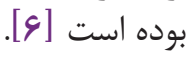
شيوع اختلالات اسكلتى ـ عضلانى در محيطهاى كارى،

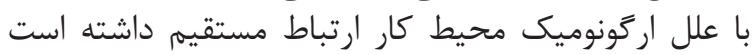

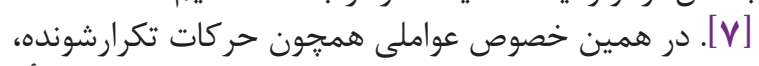

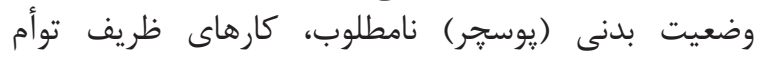

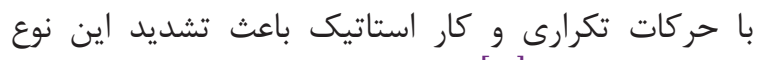

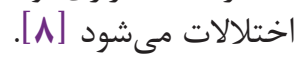

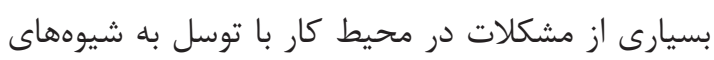

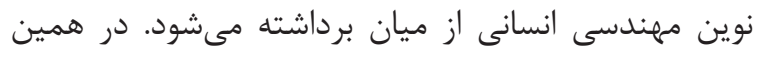

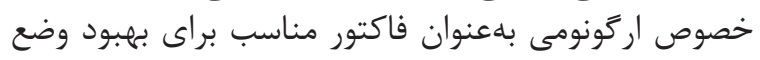

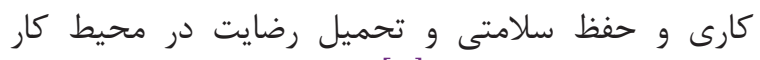

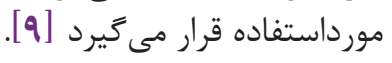
يكى از شايعترين مشكلات در ميان كارمندانى كه با مكردال
مقدمه

انستيتو ملى بهداشت و ايمنى شغلى آمريكا (NIOSH)

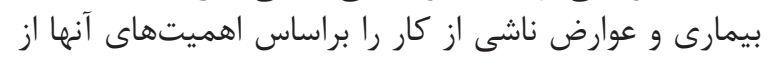

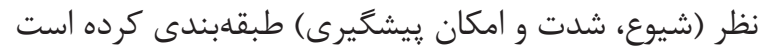

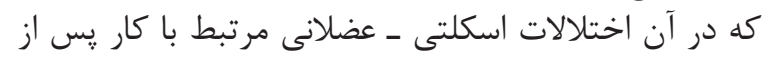

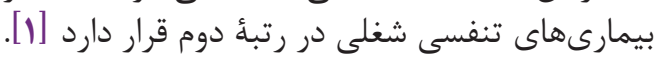

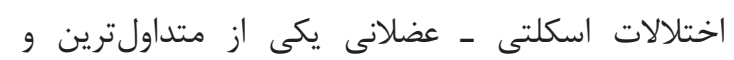

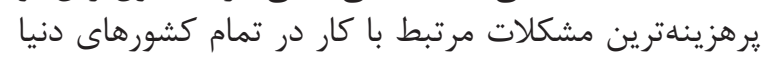

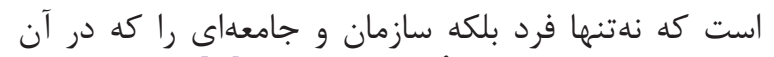

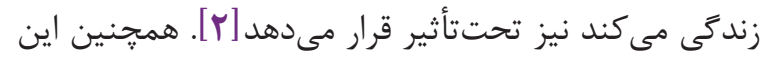

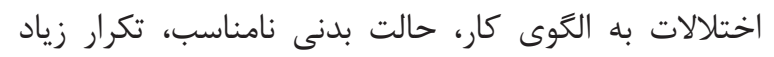

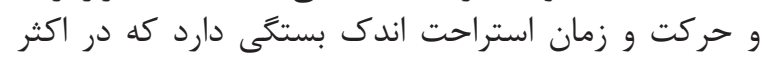

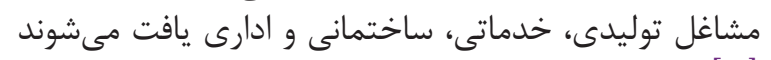

آمار نشان مى دهد شيوع و بروز اختلالات اسكلتى ـ عضلانى

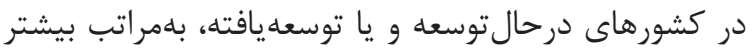

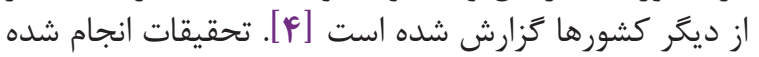


حداقل دو سال سابقع كارى، تكميل و تأييد فرم رضايت

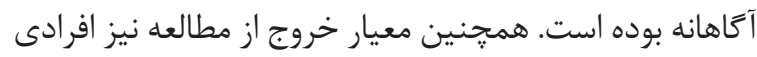
بودند كه بهدليل هركونه حادثه دجار اختلالات خدات اسكلتى عضلانى شده بودند.

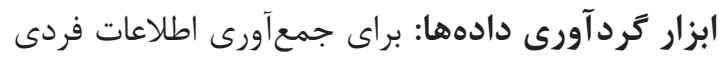

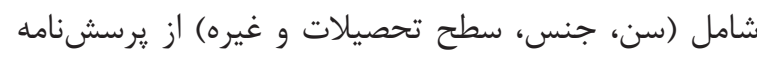

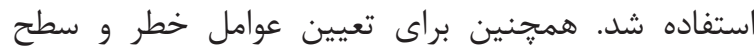

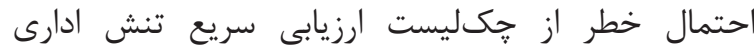

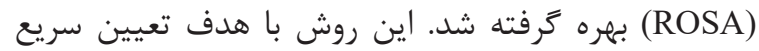

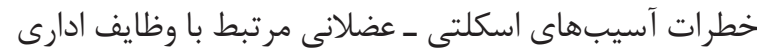

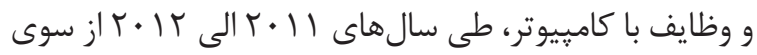
Sonne

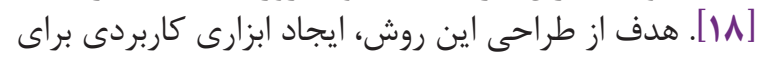

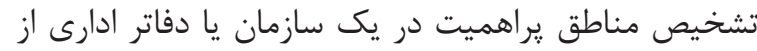

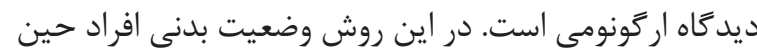

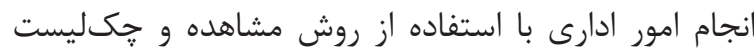

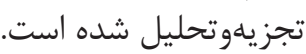

روش ارزيابى سريع تنش ادارى (ROSA) با توسعة

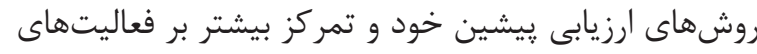

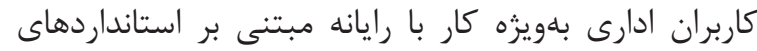
CSA standard Z412

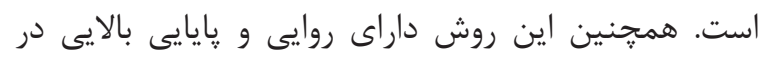

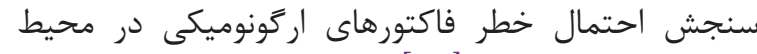
ادارى كار با رايانه است [11].

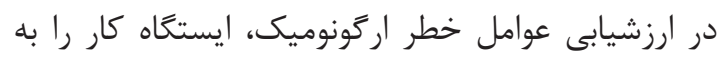

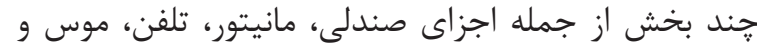

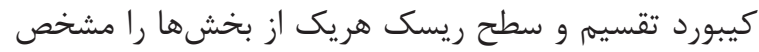

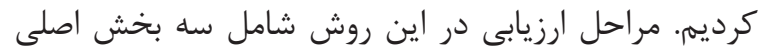

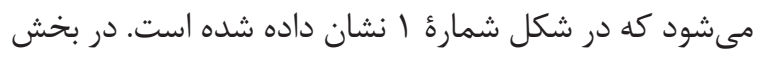

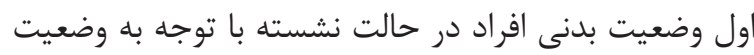

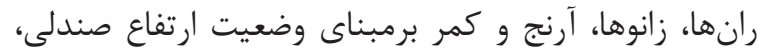

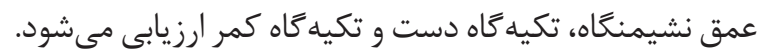

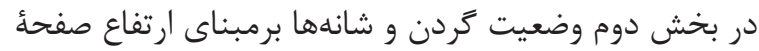

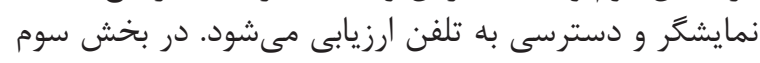

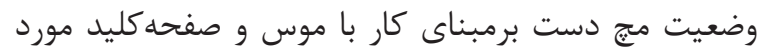

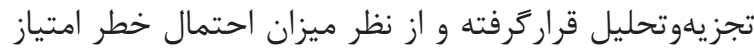

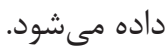

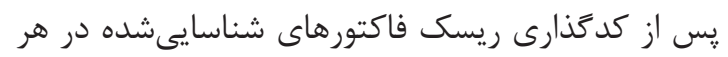

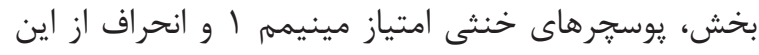

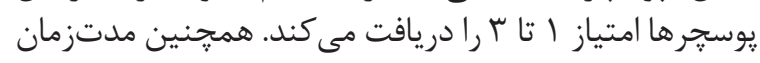

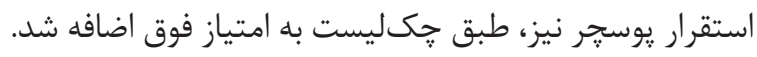

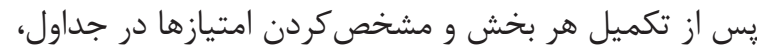

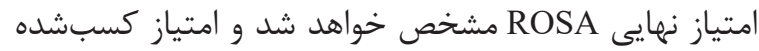

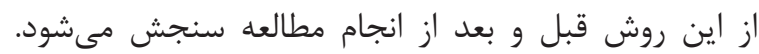

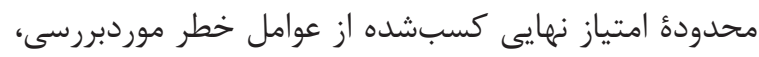

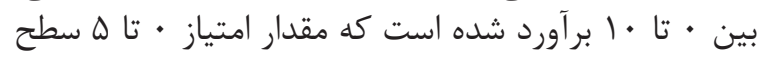

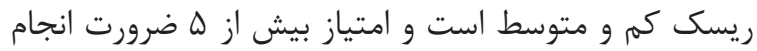

اقدام مداخلهاى و تحقيقات دقيقتر را نشان مى دهد إند [19].
كامبيوتر كار مى كنند و از علل اصلى دلايل غيبت از كار و و

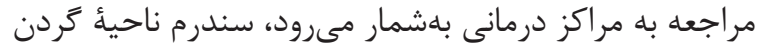

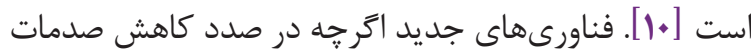

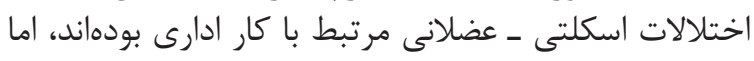

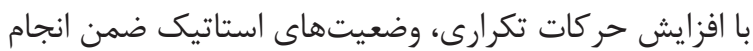

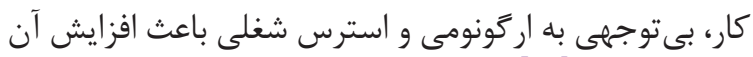

نيز شده است [11].

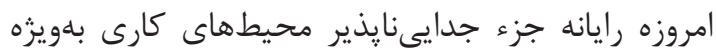

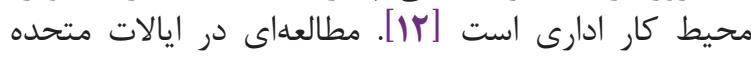

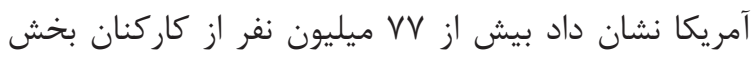

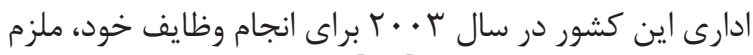

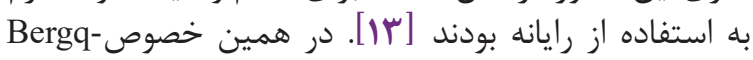

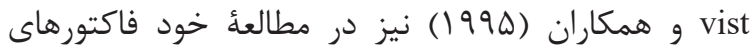

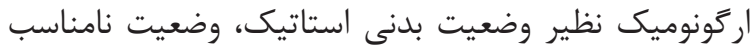

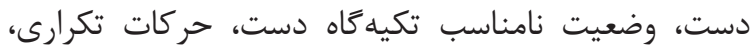
محل نامناسب استقرار نمايشكر و صفحه كليد را دان در شيوع نكان آسيبهاى اسكلتى ـ عضلانى مؤثر دانستهاند [If [If].

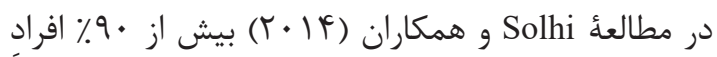

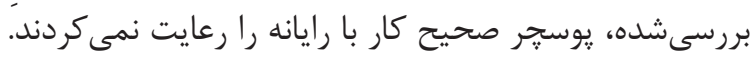

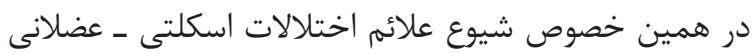

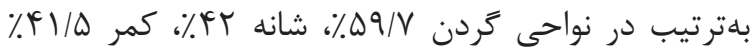

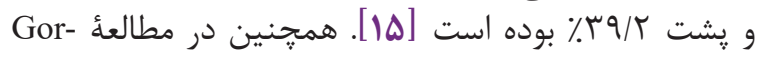

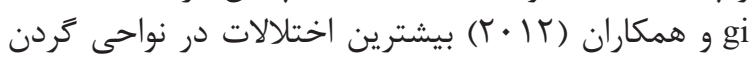

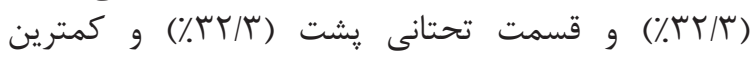

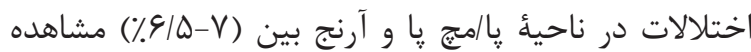

شد [19]. - اخدر

با توجه به عوامل اركونوميكى مؤثر در بروز اختلالات

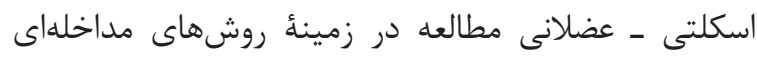

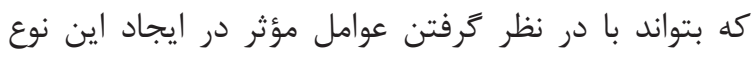

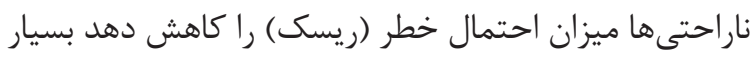

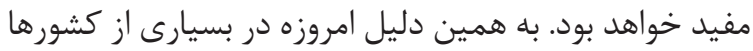

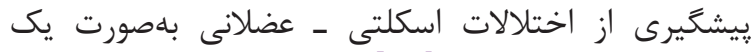
اولويت ملى در آمده است [الات اسكلتي

با عنايت به مطالب فوق و با توجه به كسترهٔ وسيع اين

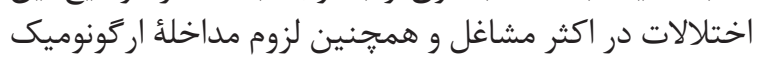

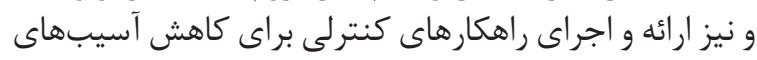

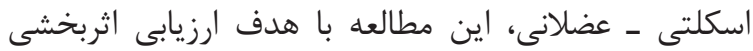

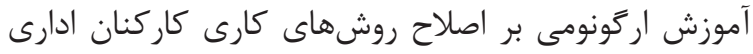

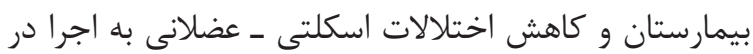

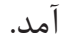

\section{مواد و روشها}

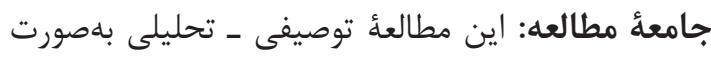

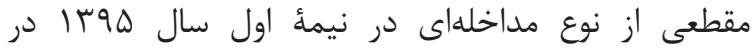

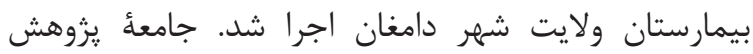

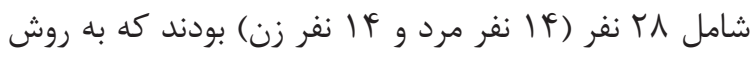

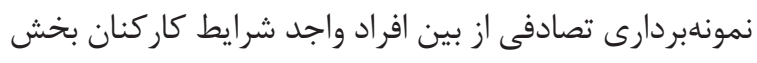
ادارى بيمارستان انتخاب شدند. معيار ورود به مطالعه دارئ داشتن 
كارى مناسب، معرفى اختلالات اسكلتى ـ عضلانى محيط

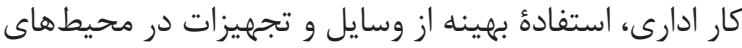

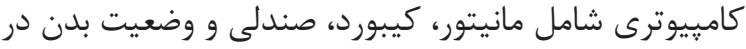

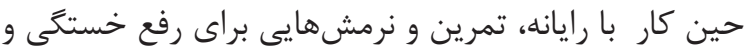

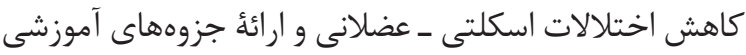

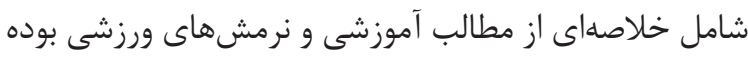

يس از 9 ماه و بعد از اجراى كامل مداخلات، بار ديخر

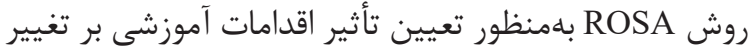
رفتار ارگونوميكى افراد حين كار استفاده شد. بعد از انجان انجام

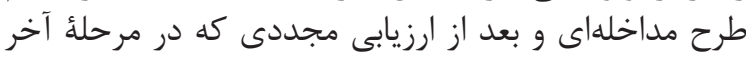

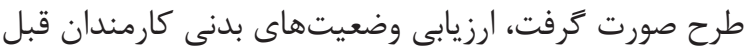

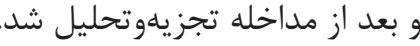

براى تجزيهوتحليل اطلاعات جمعآورىشده از نرمافزار

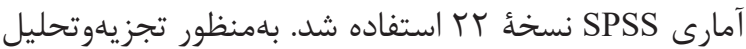

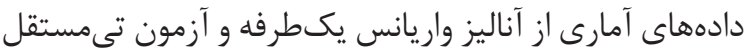

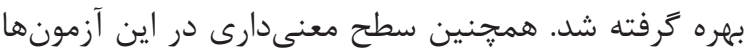

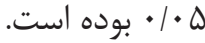

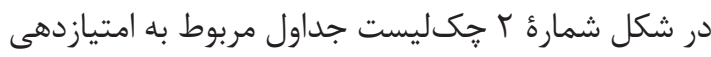

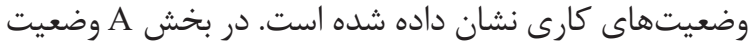

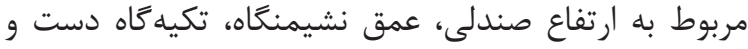

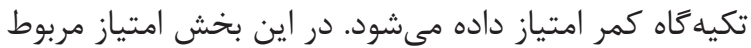

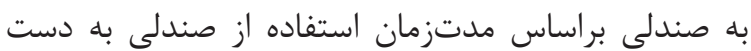

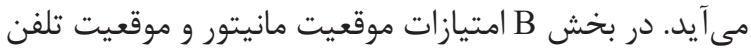

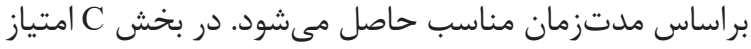

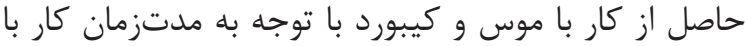

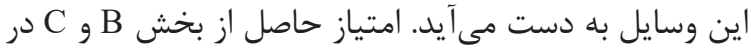

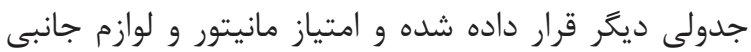

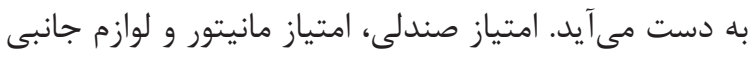

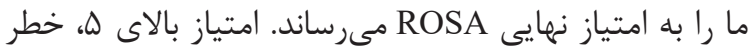

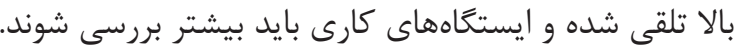
اجراى مداخلة آموزشى: يس از بررسى انجامشده و

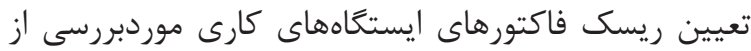

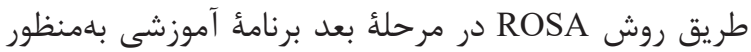

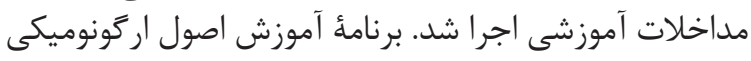

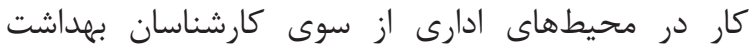

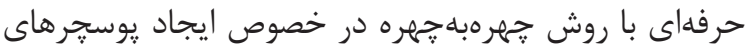
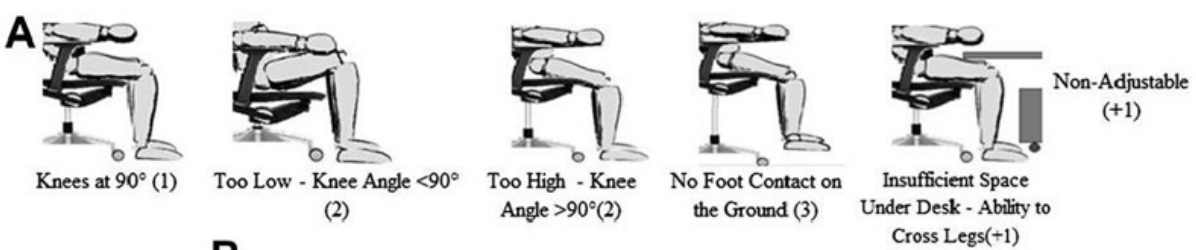

B
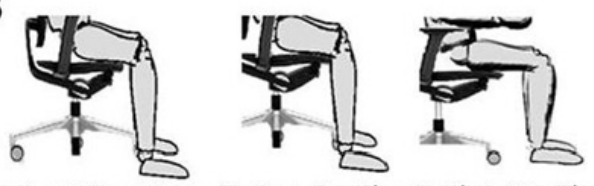

Cross Legs $(+1)$

\section{Adjustable \\ $(+1)$}

Approximately $8 \mathrm{~cm}$ of Space Too Long - Less Than Too Short - More Than Between Knee and Edge of $\quad 8 \mathrm{~cm}$ of Space (2) $\quad 8 \mathrm{~cm}$ of Space (2) Seat (1)

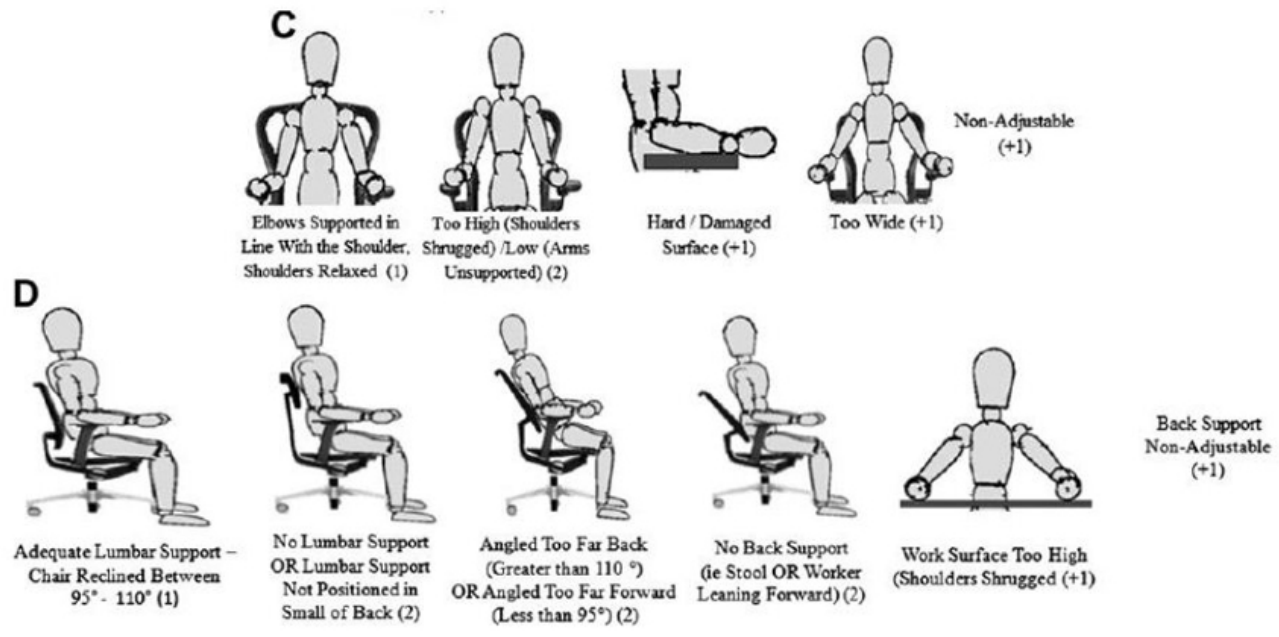

شكل ا. بخش اول؛ وضعيت بدنى افراد در حالت نشسته با توجه به وضعيت رانها، زانوها، آرنج و كمر برمبناى وضعيت

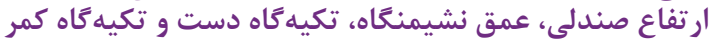




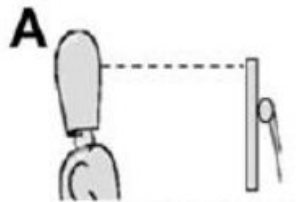

Arm's Length Distance

$(40-75 \mathrm{~cm}) /$ Screen at

Eye Level (1)

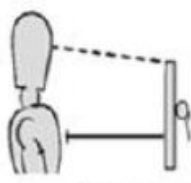

Too Low Too Far $(+1)$

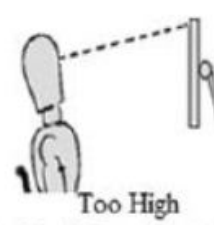

Too High
(2) (Neck Extension) (3)

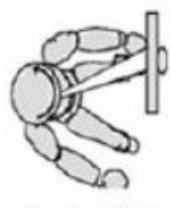

Neck Twisted

$(+1)$

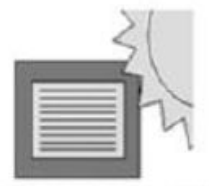

Glare on Screen $(+1)$ Documents No Holder But Required (+1)

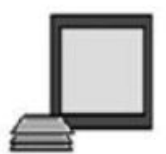<smiles>[B]C1CCCCC1C(C)C</smiles>

Headset / One Hand Too Far of Reach (Outside of $30 \mathrm{~cm}$ ) (2) Neck and Shoulder on Phone \& Neutral

Neck Posture (1)

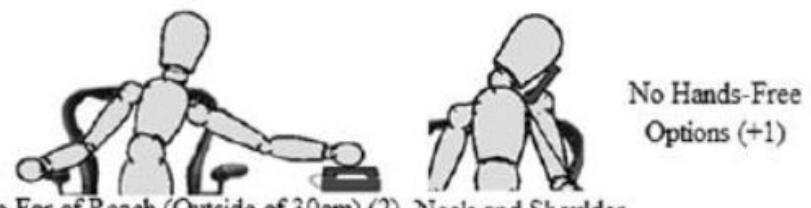

No Hands-Free

Hold (+2)

شكل ا. بخش دوم؛ امتيازات موقعيت مانيتور و موقعيت تلفن

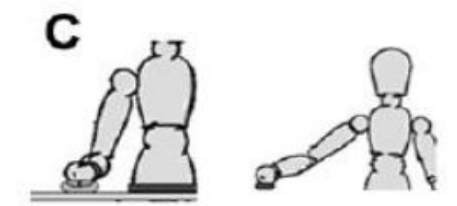

Mouse in Line with Reaching to Mouse (2) Shoulder (1)

D

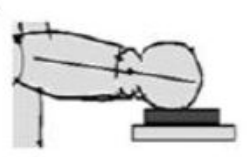

Wrists Straight, Shoulders Relaxed (1)

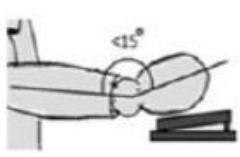

Wrists Extended Keyboard on Positive Angle $\left(>15^{\circ}\right.$ Wrist Extension) (2)

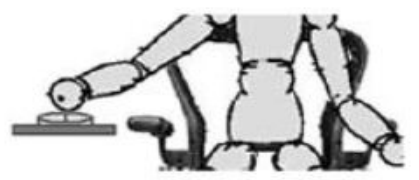

Mouse/Keyboard on Different Surfaces $(+2)$

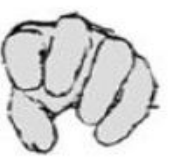

Pinch Grip on Mouse

$(+1)$

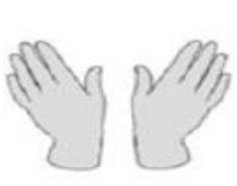

Deviation While

Typing (+1)

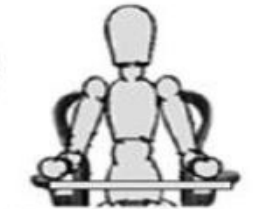

Keyboard Too High Shoulders Shrugged $(+1)$

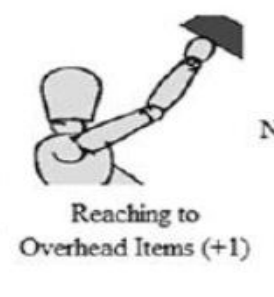

Platform

Non-Adjustable

(+1)

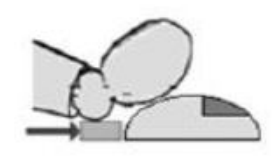

Palmrest in Front of Mouse (+1)

$$
\text { شكل ا. بخش سوم؛ امتياز حاصل از كار با موس و كيبورد }
$$
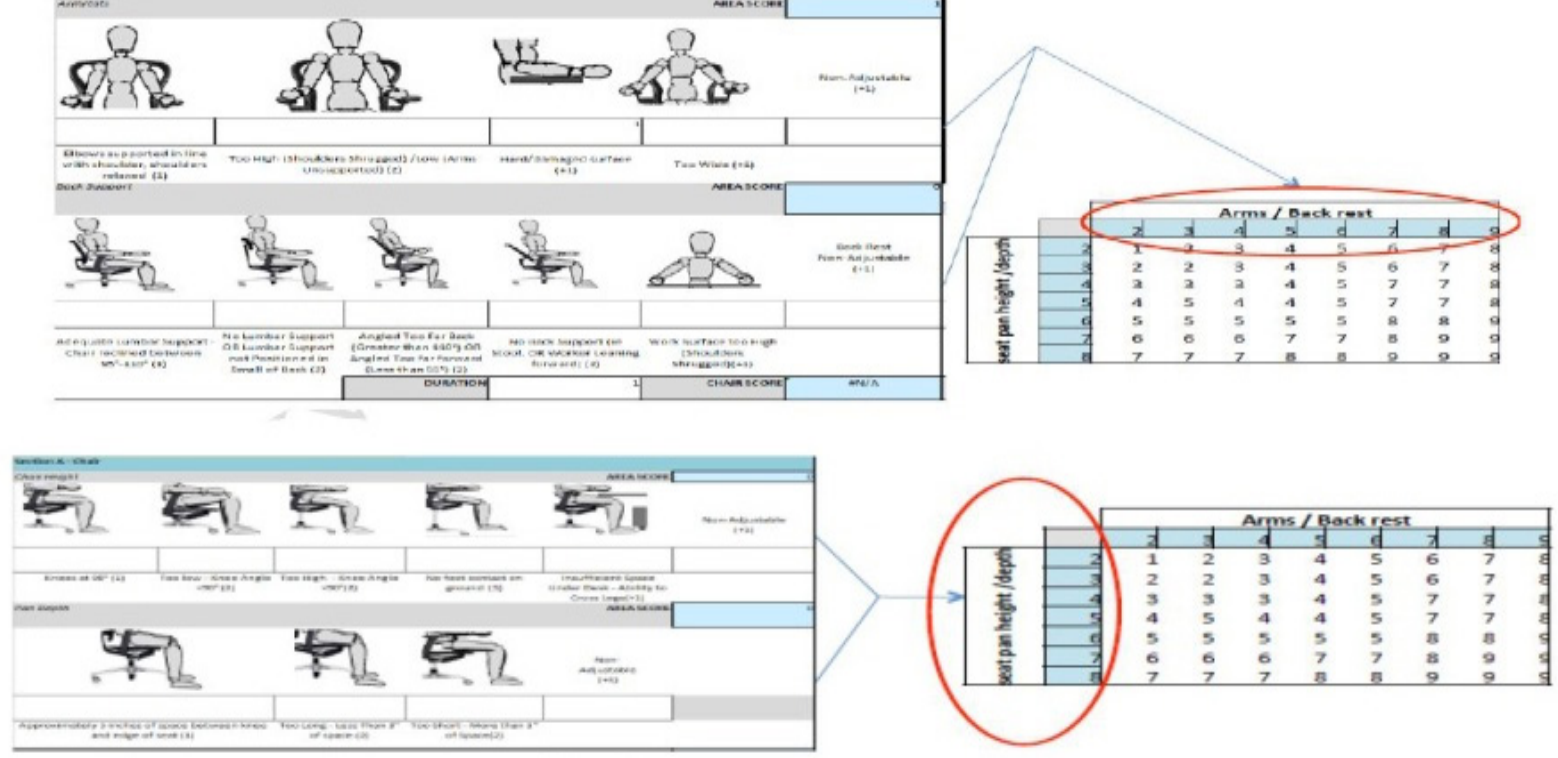

شكل r. بخش A؛ وضعيت مربوط به ار تفاع صندلى، عمق نشيمنگًاه، تكيه كاه دست و تكيه 


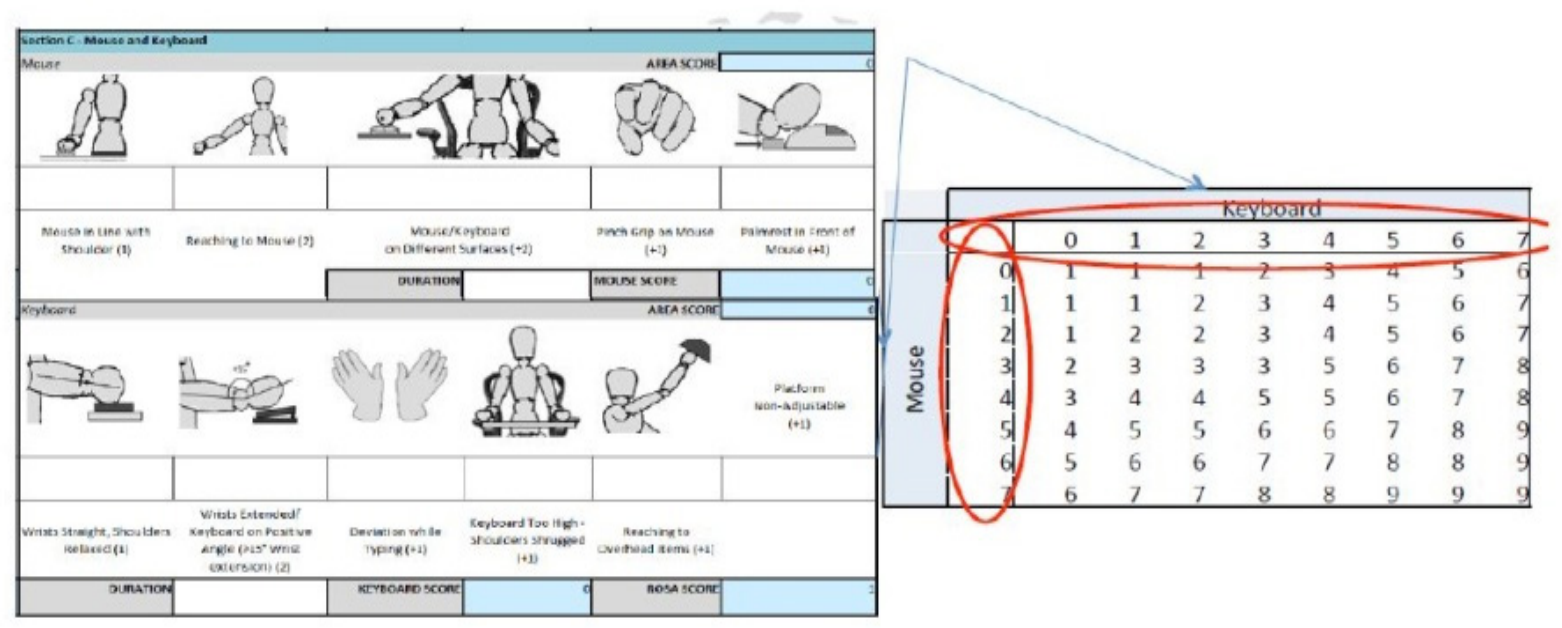

شكل r. بخش B؛ امتيازات موقعيت مانيتور و موقعيت تلفن

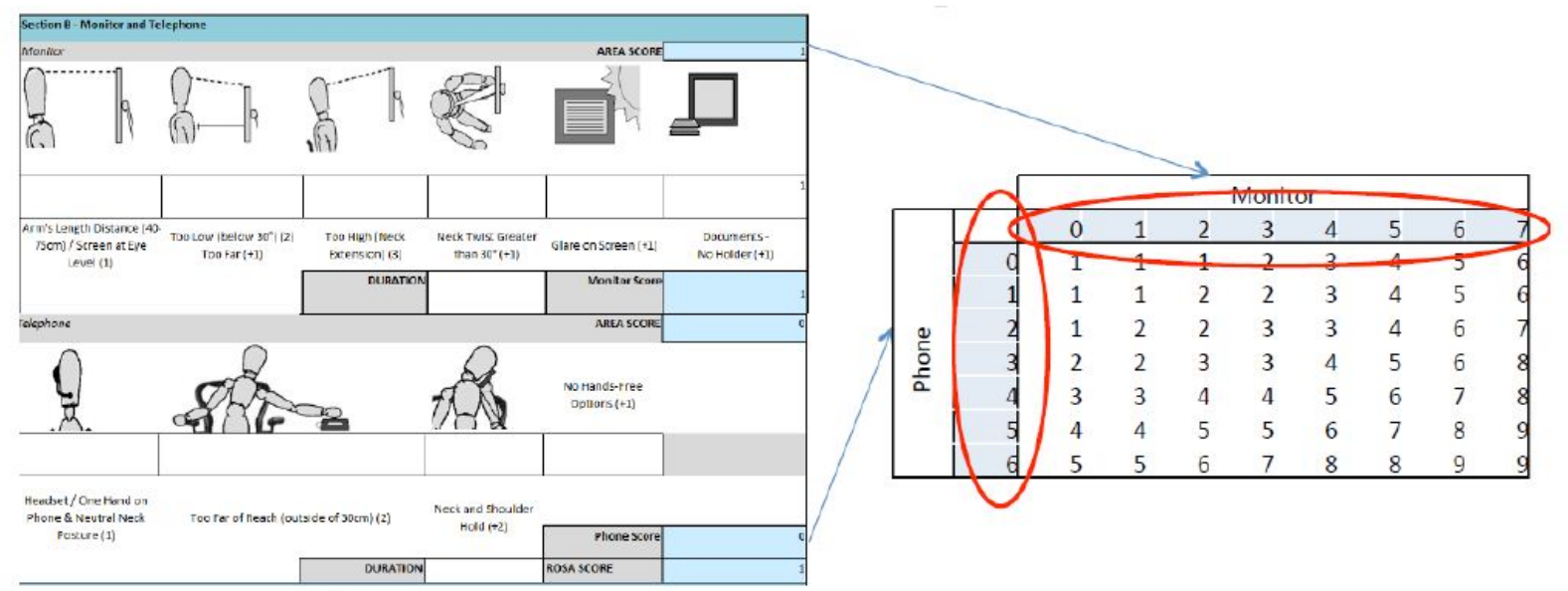

شكل r. بخش C؛ امتيازات كار با موس و كيبورد

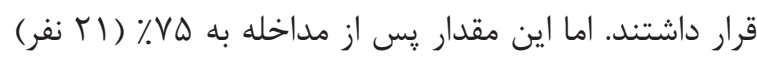

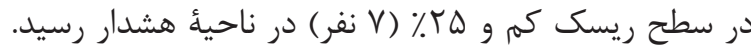

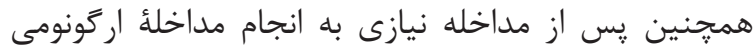
نبود.

در جدول شماره ب ميانگين امتيازهاى كسبشده سطوح

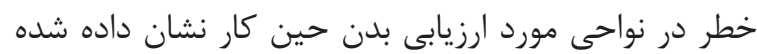

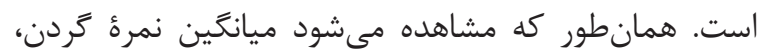

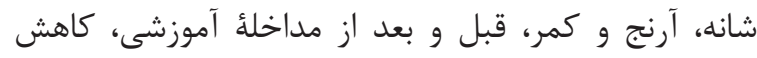

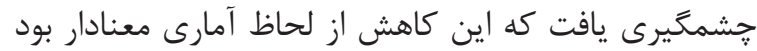

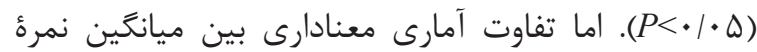

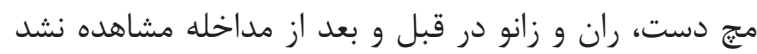

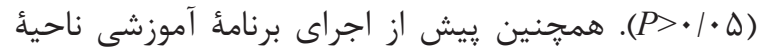

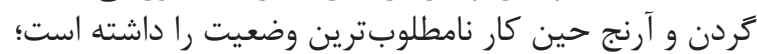

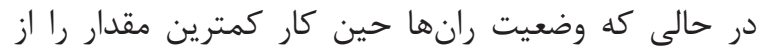

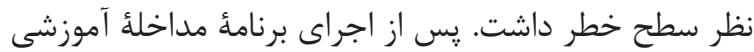

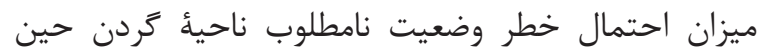

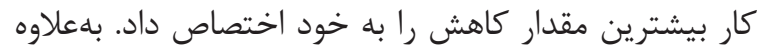

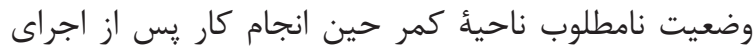
مداخله آموزشى به كمترين سطح خطر ممكن رسيد.
بافتهها

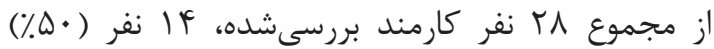

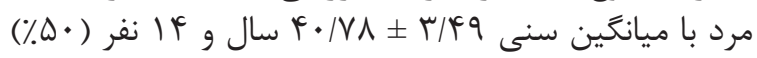

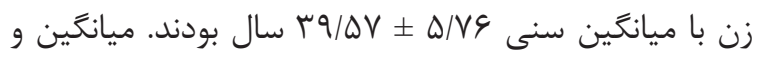

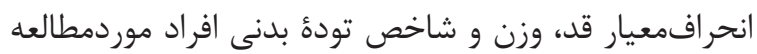

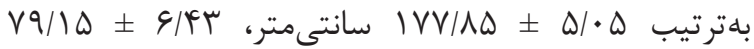
كيلو تحصيلات شركت كنند

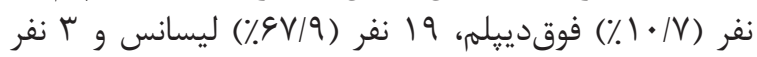
فوق ليسانس بود. سابقه شغلى افراد حداقل ه سال

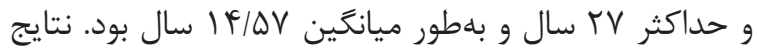

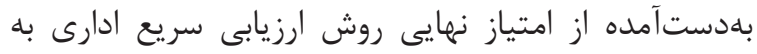

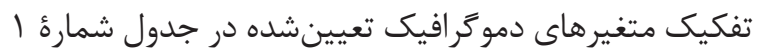
نمايش داده شده است.

سطح ريسك امتياز نهايى روش ارزيابى سريع ادارى (ROSA)

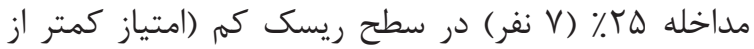

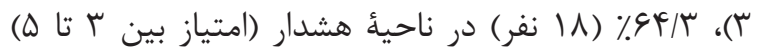

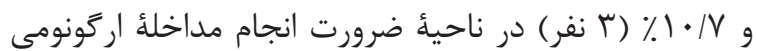


جدول ا. نتايج روش سريع تنش كاركنان ادارى شركت كنندكان (N=rA)

\begin{tabular}{|c|c|c|c|c|c|c|}
\hline \multicolumn{7}{|c|}{ ROSA امتياز نهايى } \\
\hline \multirow{2}{*}{$P$-value } & ميانتين امتياز & 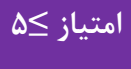 & & 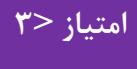 & & \multirow{2}{*}{ متغير دموكرافيك } \\
\hline & ROSA & (\%) تعداد & (\%) تعداد & (\%) تعداد & & \\
\hline \multirow{3}{*}{$<\cdot / \cdot r \Lambda$} & $r / \Lambda \Lambda \pm 1 / 9 V$ & $I(I T / \Delta)$ & $\Gamma(\Gamma V / \Delta)$ & $F(\Delta \cdot)$ & qr تا F سال (N=人) & \\
\hline & $r / 9 \Delta \pm 1 / \Delta r$ & $1(V / I)$ & $9(\xi \uparrow / \Gamma)$ & $f(Y \wedge / \varphi)$ & هr تا • & سن \\
\hline & $9 / \cdot 1 \pm 1 / 49$ & $f(\varphi q \mid 9)$ & $r(r / T)$ & - & أ تا \& & \\
\hline \multirow{2}{*}{$<\cdot 1 \cdot 10$} & $r / \varphi \cdot \pm r / \cdot \Lambda$ & $1(V / T)$ & $\Lambda(\Delta V / I)$ & $\Delta(r \Delta / V)$ & مرد (N=lf) & جنسيت \\
\hline & $V / r r \pm 1 / r q$ & $V(\Delta \cdot)$ & $f(Y \wedge / \varphi)$ & $r(Y \backslash / Y)$ & زن (N=|f) & \\
\hline \multirow{3}{*}{$<\cdot / \cdot \uparrow$. } & $r / V I \pm 1 / \Delta r$ & $I(\mathbb{f} / \Lambda)$ & $q(\Delta \cdot)$ & $\Lambda(\Upsilon \wedge / 1)$ & نرمال (N=|人) & شاخص تودهٔ بدنى \\
\hline & $\mathbb{F} / \Lambda \varepsilon \pm 1 / \mathcal{F}$ & $r(Y \wedge / 9)$ & $f(\Delta V / I)$ & $\mid(\mid f / r)$ & اضافهوزن (N=V) & \\
\hline & $9 / V \cdot \pm 1 / 99$ & $r(1 \cdots)$ & - & - & جاق (r=r) & \\
\hline \multirow{4}{*}{$<\cdot 1 \cdot p V$} & $r / l \vee \pm 1 / 11$ & - & I & $r(\varphi q \mid \varphi)$ & دييلم (N=r) & \\
\hline & $r / \Delta \pm 1 / 1 r$ & - & $r(1 \cdots)$ & - & فوقدييله (r=r) & سطح تحصيلات \\
\hline & $\Delta / V r \pm r / \cdot q$ & $\varphi(\Gamma) / \varphi)$ & $\|(\Delta V / q)$ & $r(1 \cdot / Q)$ & ليسانس (N=19) & \\
\hline & G/N1 & $r(99 \mid 9)$ & (س/ & - & فوقليسانس (r=r) & \\
\hline \multirow{3}{*}{$<\cdot / \cdot r \Delta$} & $F / \Delta F \pm|/ q|$ & $f(F \in / F)$ & (r) & $r(Y T / Y)$ & ه تا r ا سال (N=9) & \\
\hline & $9 / 11 \pm 1 / \cdot V$ & $V(|\varphi| q)$ & $\Delta(\Gamma / T)$ & $r(r \cdot)$ & rا تا •r سال (D=lه) & سابقهُ شغلى \\
\hline & $V / 0 \cdot \pm 1 / 9 F$ & $r(V Q)$ & $I(r \Delta)$ & - & ا r تا Vال (N=r) & \\
\hline
\end{tabular}

جدول r. توزيع فراوانى نمرة سطوح احتمال خطر براساس روش ROSA در افراد موردمطالعه، قبل و

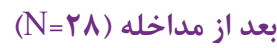

\begin{tabular}{|c|c|c|c|c|}
\hline \multicolumn{2}{|c|}{ بعد از مداخله } & \multicolumn{2}{|c|}{ قبل از مداخله } & \multirow{2}{*}{ امتياز } \\
\hline درصد & فراوانى & درصد & فراوانى & \\
\hline TQ & v & $V / I$ & r & r \\
\hline$\Delta \cdot$ & If & $1 V / 9$ & $\Delta$ & r \\
\hline $1 V / 9$ & $\Delta$ & $r q / r$ & 11 & f \\
\hline$V / I$ & r & TQ & v & $\Delta$ \\
\hline . & · & $V / I$ & r & $\varepsilon$ \\
\hline · & · & r/q & 1 & v \\
\hline
\end{tabular}


جدول س. ميانغين امتيازهاى ROSA كسبشده براى وضعيتهاى بدنى كارى مطالعهشده قبل و بعد

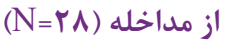

\begin{tabular}{|c|c|c|c|c|c|}
\hline \multirow{2}{*}{$P$-value } & \multicolumn{2}{|c|}{ بعد از مداخله } & \multicolumn{2}{|c|}{ قبل از مداخله } & \multirow{2}{*}{ واحى موردارزيابى } \\
\hline & انحرافمعيار & ميانََين & انحرافمعيار & ميانگين & \\
\hline$\cdot 1 \cdot 99$ & $1 / \cdot V$ & $1 / 9$. & .191 & $F / T 1$ & كردن \\
\hline •|•r & $\cdot|V|$ & I/AT & $\cdot / r q$ & ऍ/^q & شانه \\
\hline $.1 \cdot 19$ & $\cdot / r \Lambda$ & I/AV & $\cdot / \mu F$ & $F / l f$ & آرنج \\
\hline .1 .90 & $1 / .9$ & $1 / \lambda$. & $1 / \cdot r$ & $r / \wedge \Delta$ & مـج دست \\
\hline$\cdot / \cdot r \Lambda$ & $1 / \cdot 1$ & $\cdot / A V$ & ( & $r / l$ & كمر \\
\hline$\cdot / 1 \cdot V$ & $1 / 11$ & $1 / \cdot r$ & $1 / 1 F$ & $r / l$. & ران \\
\hline$\cdot|| Q \mid$ & $1 / 1 r$ & $1 / 1 V$ & $\cdot / \vee 9$ & T/VA & زانو \\
\hline
\end{tabular}

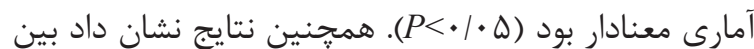

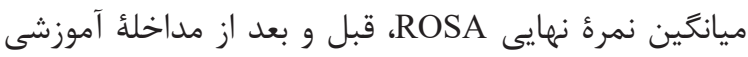

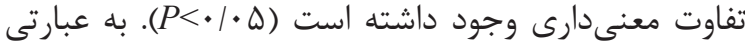

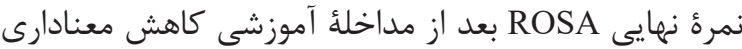

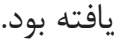

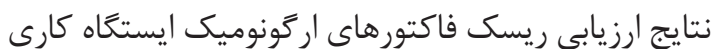

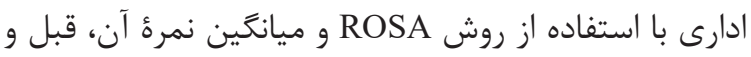

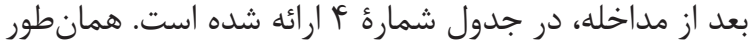

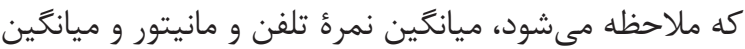

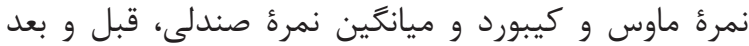

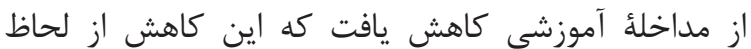

جدول f. ميانغين نمرةٔ اجزاى مختلف و نمرةٔ نهايى ROSA، قبل و بعد از مداخله (N=rA)

\begin{tabular}{|c|c|c|c|}
\hline \multirow{2}{*}{$P$-value } & بعد از مداخله & قبل از مداخله & \\
\hline & انحرافعيار 土 ميانگين & انحر افمعيار 土 ميانكين & \\
\hline$\cdot / \cdot 4$. & $r / \cdot 1 \pm \cdot / V V$ & $F / v q \pm 1 / 1 \Delta$ & تلفن و مانيتور \\
\hline $.1 \cdot 44$ & $r / I F \pm 1 / 9 V$ & $F / F \mid \pm 1 / \cdot 9$ & موس و كيبورد \\
\hline $.1 \cdot 19$ & $r / \wedge 9 \pm 1 / \Delta \Delta$ & $\Delta / r r \pm 1 / 9 \Lambda$ & صندلى \\
\hline$\cdot / \cdot r q$ & $\varphi / \cdot \Delta \pm \cdot / \cdot 9 \Lambda$ & $\Delta / \wedge \Delta \pm r / V)$ & نمرة نهايى ROSA \\
\hline
\end{tabular}

[Tr]]. به عقيده اغلب يزوهشگران، كار ادارى بهدليل ماهيت شغلى خود اغلب نيازمند ايجاد يك وضعيت استاتيك دريك در

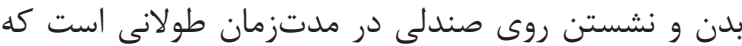

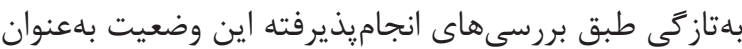

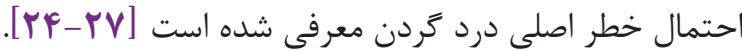

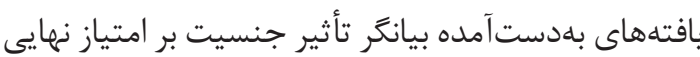

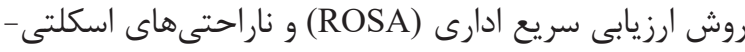

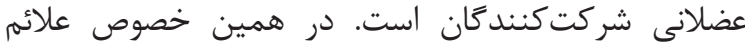

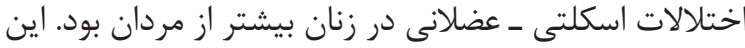

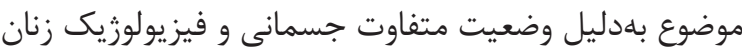

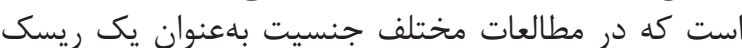

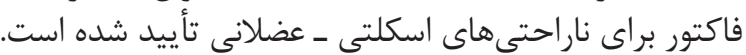

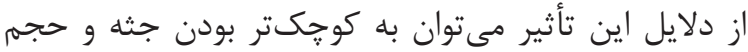

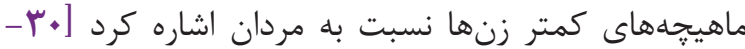

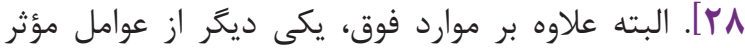

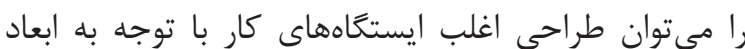

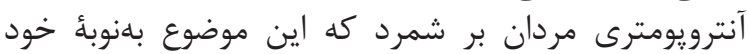

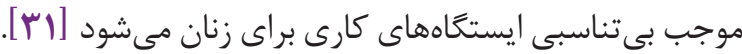

\section{بحث و نتيجه گيرى}

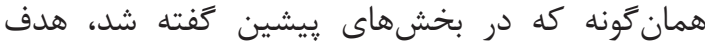

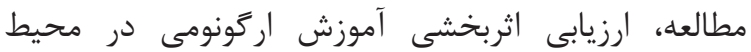

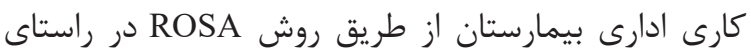

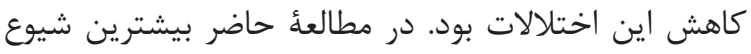

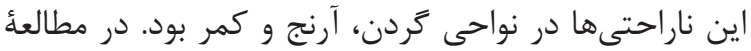
Akrouf

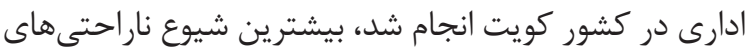

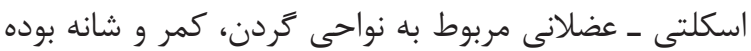

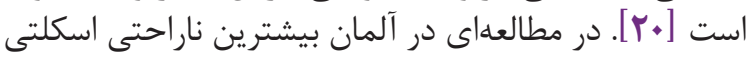

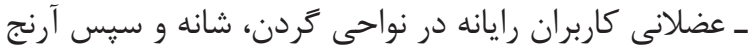

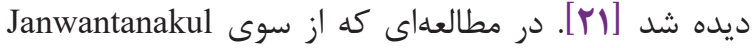

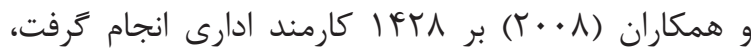

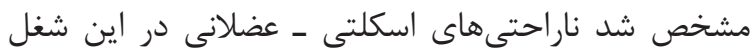

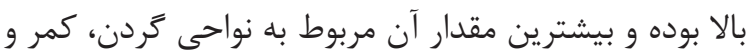

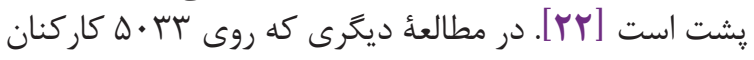

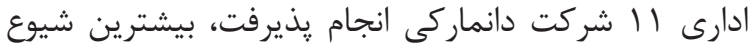

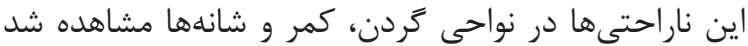




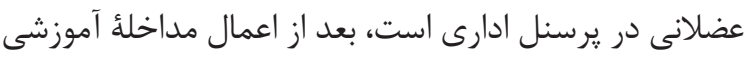

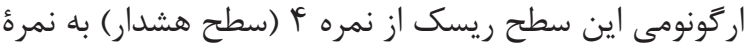

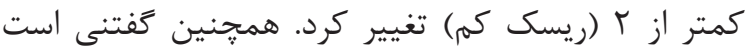

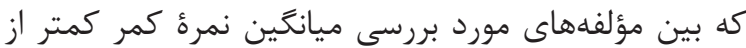

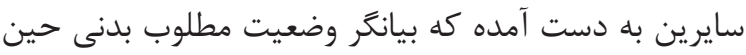

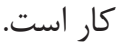

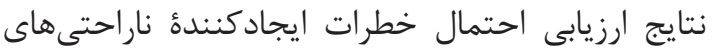

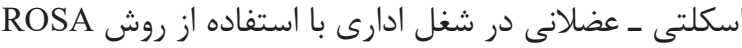

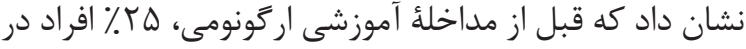

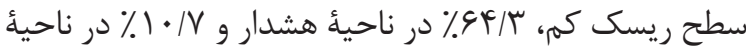

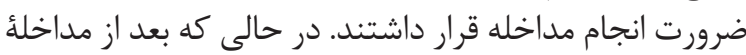

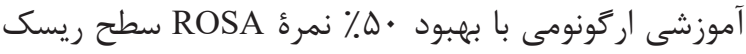

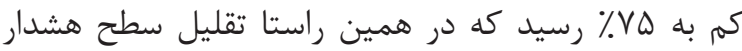

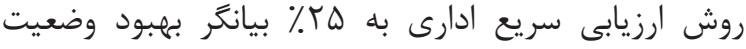

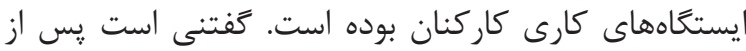

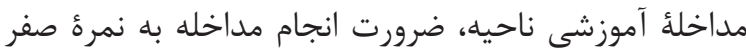

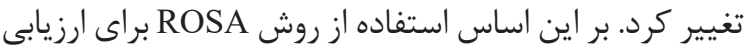

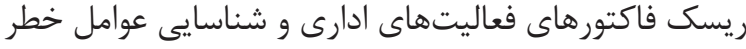

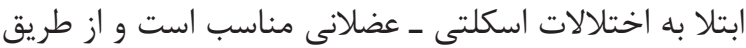

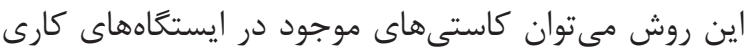

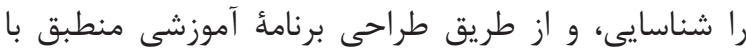

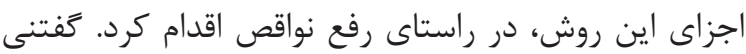

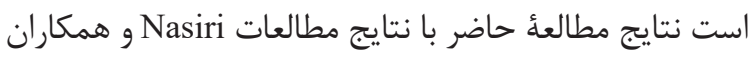

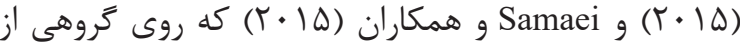

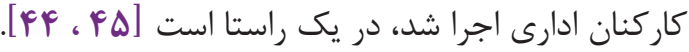
از محدوديتهاى اين مطالعه، كمبود تعداد نمونههاى در

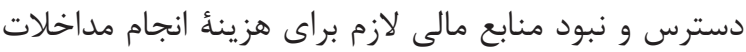

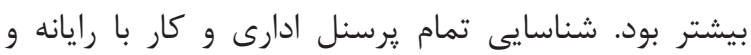

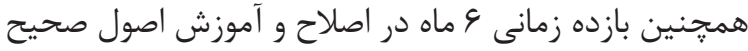
اركونومى مىتواند از نقاط قوت اين مطاه دالعه اصلاح باشد.

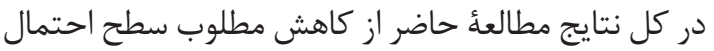

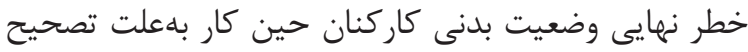

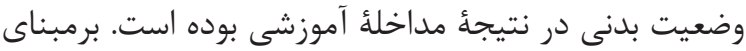

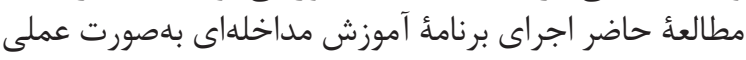

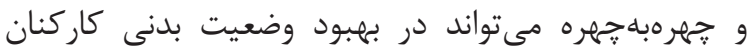

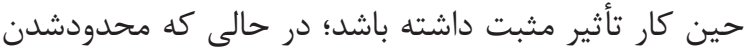

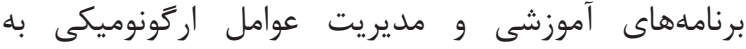

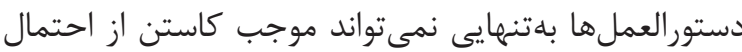
خطرات ناشى از عوامل اركونوميكى باشد نداند.

\section{سياسگزارى}

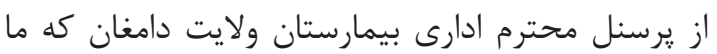
را در اين يزوهش يارى كردند، صميمانه تشكر و قدردانى مى شود.

\section{تعارض منافع}

بين نويسندكان هيجَّونه تعارضى در منافع وجود ندارد.

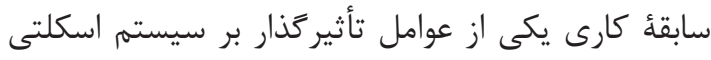

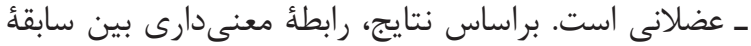

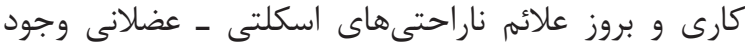

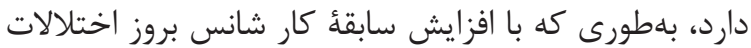

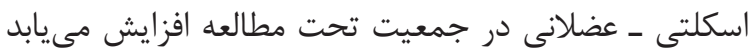

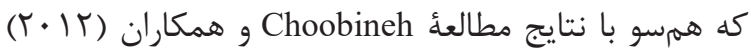

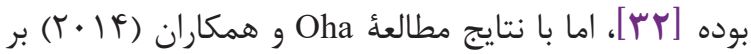

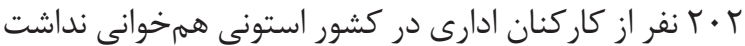

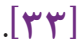

تأثير معنىدار بودن شاخص توده بدنى (BMI) بر امتياز

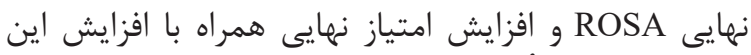

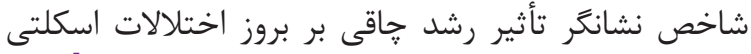

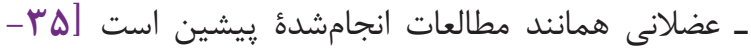

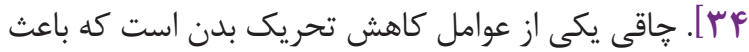

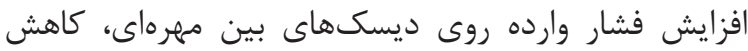

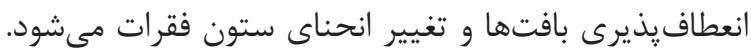

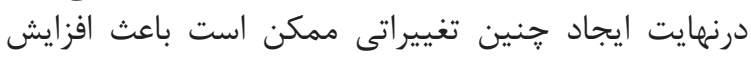

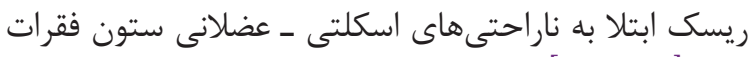

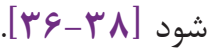

آنجه از اين مطالعه بر مى آيد اين است كه با افزايش سطح

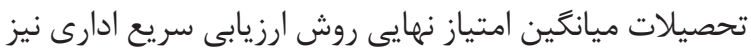

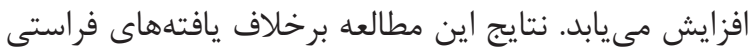

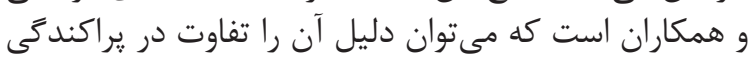

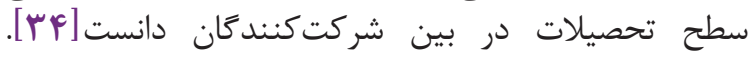

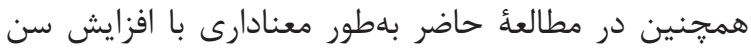

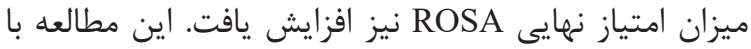

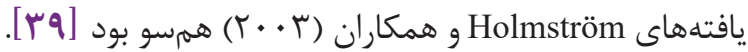
در مطالعهُ حاضر تغيير در نمرءٔ تلفن و مانيتور، موس و ود

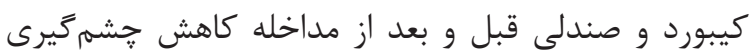

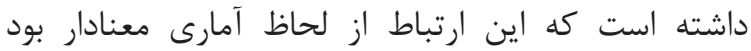

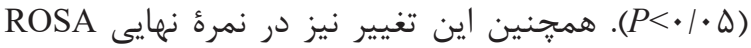

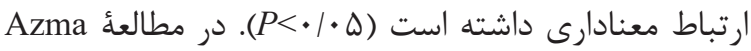

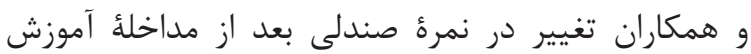

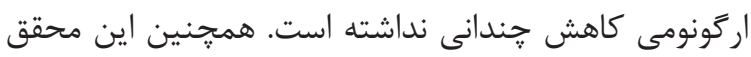

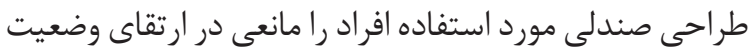

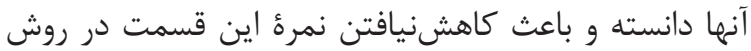

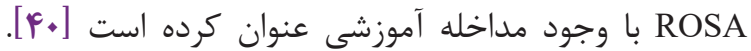

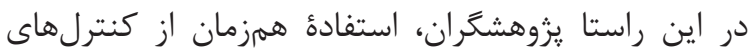

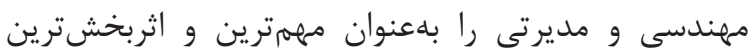

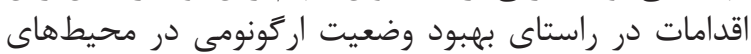

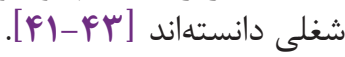

در مطالعُٔ مداخلهاى حاضر تصحيح وضعيت بدن هنئام

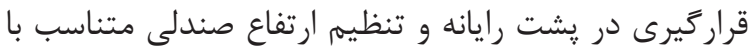

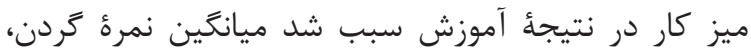

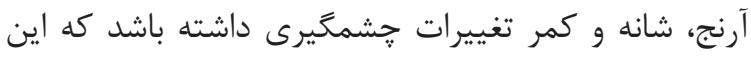

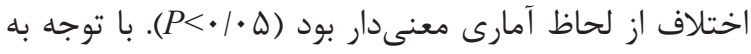

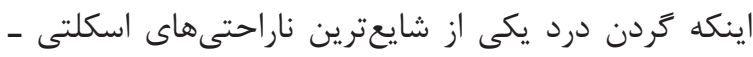




\section{References}

1. Tayyari F, Smith JL. Occupational ergonomics: Principles and applications (Manufacturing systems engineering series). United Kingdom: Chapman \& Hall London; 1997.

2. Kathy Cheng HY, Cheng CY, Ju YY. Work-related musculoskeletal disorders and ergonomic risk factors in early intervention educators. Appl Ergon. 2012; 44(1): 134-41.https://doi.org/10.1016/j. apergo.2012.06.004 PMID:22770544

3. Devereux J, Vlachonikolis I, Buckle P. Epidemiological study to investigate potential interaction between physical and psychosocial factors at work that may increase the risk of symptoms of musculoskeletal disorder of the neck and upper limb. Occupational and environmental medicine. 2002;59(4):269-77.

4. Waters TR, Dick RB, Krieg EF. Trends in work-related musculoskeletal disorders: a comparison of risk factors for symptoms using quality of work life data from the 2002 and 2006 general social survey. J Occup Environ Med. 2011; 53(9): 1013-24.https://doi.org/10.1097/JOM.0b013e3181fc8493 PMID:21278598

5. Vanwonterghem K. Work-related musculoskeletal problems: Some ergonomic considerations. Journal of human ergology. 1996;25(1):5-13

6. Hasanzadeh M. Review and determine the risk factors causing musculoskeletal disorders among the buoys staffs (Doctoral dissertation, MS Thesis in Occupational Health], Tehran, School of public health, Tehran university of medical sciences. 2001.

7. Choobineh A, Tabatabaei SH, Mokhtarzadeh A, Salehi M. Musculoskeletal problems among workers of an Iranian rubber factory. J Occup Health. 2007; 49(5): 418-23.https://doi. org/10.1539/joh.49.418. PMID: 17951976

8. 1.Lorusso A, Bruno S, L'Abbate N. A review of low back pain and musculoskeletal disorders among Italian nursing personnel. Ind Health. 2007;45(5):637-44.https://doi.org/10.2486/indhealth.45.637 PMID: 18057806

9. Das B, Sengupta AK. Industrial workstation design: a systematic ergonomics approach. Applied Ergonomics.1996; 27(3): 157-63.https://doi. org/10.1016/0003-6870(96)00008-7

10. Andersen JH, Harhoff M, Grimstrup S, Vilstrup I, Lassen CF, Brandt LP, et al. Computer mouse use predicts acute pain but not prolonged or chronic pain in the neck and shoulder. Occup Environ Med. 2008; 65(2): 126-31.https://doi. org/10.1136/oem.2007.033506 PMID: 17681996

11. Kraatz S, Lang J, Kraus T, Münster E, Ochsmann E. The incremental effect of psychosocial workplace factors on the development of neck and shoulder disorders: a systematic review of longitudinal studies. Int Arch Occup Environ Health. 2013; 86(4): 375-95.https://doi.org/10.1007/ s00420-013-0848-y. PMID:23549669

12. Shariat A, Cleland JA, Danaee M, Kargarfard M, Sangelaji B, Tamrin SB. Effects of stretching exercise training and ergonomic modifications on musculoskeletal discomforts of office workers: a randomized controlled trial. Braz J Phys Ther. 2018; 22(2): 144-53.https://doi. org/10.1016/j.bjpt.2017.09.003 PMID:28939263 PMCID:PMC5883995

13. IJmker S, Blatter BM, Van der Beek AJ, Van Mechelen W, Bongers PM. Prospective research on musculoskeletal disorders in office workers (PROMO): study protocol. BMC musculoskeletal disorders. 2006; 7(5): 1-9. https://doi. org/10.1186/1471-2474-7-55

14. Bergqvist U, Wolgast E. Nilsson B. Musculoskeletal disorders among visual display terminal workers: individual, ergonomic, and work organizational factors. Ergonomics. 1995; 38(4): 76376.https://doi.org/10.1080/00140139508925148 PMID: $\underline{7729403}$

15. Solhi M, Khalili Z, Zakerian SA, Eshraghian MR. Prevalence of symptom of musculoskeletal disorders and predictors of proper posture among computer users based on stages of change model in computer users in central Headquarter, Tehran University of Medical Sciences. Iran Occupational Health. 2014;11(5):43-52.

16. Gorgi Z, Assadollahi Z, Ghaffarian A, Rezaeian M. The Prevalence of Musculoskeletal Disorders in the Employees of Office Systems at Rafsanjan University of Medical Sciences in 2012. Journal 
of Rafsanjan University of Medical Sciences. 2014 Mar 15;12(12):991-1002.

17. Spielholz P, Silverstein B, Morgan M, Checkoway H, Kaufman J. Comparison of self-report, video observation and direct measurement methods for upper extremity musculoskeletal disorder physical risk factors. Ergonomics. 2001;44(6):588613.https://doi.org/10.1080/00140130118050 PMID: 11373023

18. Andrews DM. The Rapid Office Strain Assessment (ROSA): Validity of online worker self-assessments and the relationship to worker discomfort. Occupational Ergonomics. 2011 Jan $1 ; 10(3): 83-101$.

19. Sonne M, Villalta DL, Andrews DM. Development and evaluation of an office ergonomic risk checklist: ROSA Rapid office strain assessment. Appl Ergon. 2012; 43(1): 98-108. https://doi.org/10.1016/j.apergo.2011.03.008 PMid:21529772

20. Akrouf QA, Crawford JO, Al Shatti AS, Kamel MI. Musculoskeletal disorders among bank office workers in Kuwait. East Mediterr Health J. 2010; 16(1): 94-100.https://doi. org/10.26719/2010.16.1.94 PMID:20214165

21. Rempel DM, Krause N, Goldberg R. A randomized controlled trial evaluating the effects of two work station interventions on upper body pain and incident musculoskeletal disorders among computer operators. Occup Environ Med. 2006; 63(5): 300-6.https://doi. org/10.1136/oem.2005.022285 PMID: 16621849 PMCID:PMC2092482

22. Janwantanakul P, Pensri P, Jiamjarasrangsri V, Sinsongsook T. Prevalence of self-reported musculo-skeletal symptoms among office workers. Occup Med (Lond). 2008; 58(6): 436-8.https://doi.org/10.1093/occmed/kqn072 PMID: $\underline{18544589}$

23. Juul-Kristensen B, Jensen C. Self-reported workplace related ergonomic conditions as prognostic fac-tors for musculoskeletal symptoms: the "BIT" follow up study on office workers. Occup Environ Med. 2005; 62(3): 188-94.https://doi. org/10.1136/oem.2004.013920 PMID:15723884 PMCID:PMC1740969

24. Jun D, Zoe M, Johnston V, O’Leary S. Physical risk factors for developing non-specific neck pain in office workers: a systematic review and meta-analysis. Int Arch Occup Environ Health. 2017; 90(5): 373-410.https://doi.org/10.1007/ s00420-017-1205-3 PMID:28224291

25. Cho CY, Hwang YS, Cherng RJ. Musculoskeletal symptoms and associated risk factors among office workers with high workload computer use. J Manipulative Physiol Ther. 2012; 35(7): 53440.https://doi.org/10.1016/i.jmpt.2012.07.004 PMID:22951267

26. Côté P, van der Velde G, Cassidy JD, Carroll LJ, Hogg-Johnson S, Holm LW, Carragee EJ, Haldeman S, Nordin M, Hurwitz EL, Guzman J. The burden and determinants of neck pain in workers: results of the Bone and Joint Decade 2000-2010 Task Force on Neck Pain and Its Associated Disorders. Journal of manipulative and physiological therapeutics. 2009; 32(2): S7086.https://doi.org/10.1016/j.jmpt.2008.11.012 PMID: 19251078

27. Gane EM, Michaleff ZA, Cottrell MA, McPhail SM, Hatton AL, Panizza BJ, et al. Prevalence, incidence, and risk factors for shoulder and neck dysfunction after neck dissection: a systematic review. EJSO. 2017; 43(7): 1199-218.https://doi. org/10.1016/j.ejso.2016.10.026 PMID:27956321

28. Wu S, He L, Li J, Wang J, Wang1 S. Visual display terminal use increases the prevalence and risk of workrelated musculoskeletal disorders among Chinese office workers: a cross-sectional study. Journal of ocuppational health. 2012; 54(1): 3443.https://doi.org/10.1539/joh.11-0119-OA

29. Robertson MM, Ciriello VM, Garabet AM. Office ergonomics training and a sit-stand workstation: Effects on musculoskeletal and visual symptoms and performance of office workers. Applied Ergonomics. 2012; 44(1): 73-85. https://doi.org/10.1016/j.apergo.2012.05.001 PMID:22727324

30. Treaster DE, Burr D. Gender differences in prevalence of upper extremity musculoskeletal disorders. Ergonomics. 2004; 47(5): 495-526. https://doi.org/10.1080/00140130310001638171 PMID:15204301

31. Ming Z, Närhi M, Siivola J. Neck and shoulder pain related to computer use. Pathophysiolo- 
gy. 2004; 11(1): 51-6. https://doi.org/10.1016/j. pathophys.2004.03.001 PMID: 15177516

32. Choobineh AR, Rahimi Fard H, Jahangiri M, Mahmood Khani S. Musculoskeletal injuries and their associated risk factors. Iran Occupational Health. 2012;8(4):70-81.

33. Oha K, Animägi L, Pääsuke M, Coggon D, Merisalu E. Individual and work-related risk factors for musculoskeletal pain: a cross-sectional study among Estonian computer users. BMC musculoskeletal disorders. 2014; 15(1): 181. https://doi. org/10.1186/1471-2474-15-181 PMID:24884911 PMCID:PMC4049436

34. Ferasati F, Jalilian M. Evaluation of WMSDs in VDT users with Rapid office strain assessment (ROSA) method. Journal of Ergonomics. 2014;1(3):65-74.

35. Lee SY, Gallagher D. Assessment methods in human body composition. Current opinion in clinical nutrition and metabolic care. 2008; 11(5): 566-72. https://doi.org/10.1097/ $\underline{\text { MCO.0b013e32830b5f23 }} \quad$ PMID: 18685451 PMCID:PMC2741386

36. Meredith DS, Huang RC, Nguyen J, Lyman S. Obesity increases the risk of recurrent herniated nucleus pulposus after lumbar microdiscectomy. The Spine Journal. 2010; 10(7): 575-80. https://doi.org/10.1016/j.spinee.2010.02.021 PMID:20347400

37. Kuorinka I, Jonsson B, Kilbom A, Vinterberg H, Biering-Sorensen F, Andersson G, et al. Standardised Nordic questionnaires for the analysis of musculo-skeletal symptoms. Appl Ergon. 1987; 18(3): 233-7. https://doi.org/10.1016/00036870(87)90010-X

38. Wahlstrom J. Ergonomics, musculoskeletal disor-ders and computer work. Occup Med lnd. 2005; 55(3): 168-76. https://doi.org/10.1093/oc$\underline{\mathrm{cmed} / \mathrm{kqi} 083}$ PMID: $\underline{15857896}$

39. Holmström E, Engholm G. Musculoskeletal disorders in relation to age and occupation in Swedish construction workers. American Journal of Industrial Medicine. 2003; 44(4): 377-84. https:// doi.org/10.1002/ajim.10281 PMID:14502765

40. Nasiri IM. The Survey of Musculoskeletal Disorders Risk Factors among Office Workers and the
Implementation of an Ergonomic Training Program. Journal Mil Med. 2015 Mar 15;16(4):2116.

41. Bohr PC. Efficacy of office ergonomics education. Journal of occupational rehabilitation. 2000; 10(4): 243-55. https://doi. org/10.1023/A:1009464315358

42. Amick III BC, Robertson MM, DeRango K, Bazzani L, Moore A, Rooney T, Harrist R. Effect of office ergonomics intervention on reducing musculoskeletal symptoms. Spine. 2003; 28(24): 2706-11. https://doi.org/10.1097/01. BRS.0000099740.87791.F7 PMID:14673374

43. Liebregts J, Sonne M, Potvin JR. Photograph-based ergonomic evaluations using the Rapid Office Strain Assessment (ROSA). Appl Ergon. 2016; 52: 317-24.https://doi.org/10.1016/j. apergo.2015.07.028 PMID:26360224

44. Nasiri I, Motamedzade M, Golmohammadi R, Faradmal J. Assessment of risk factors for musculoskeletal disorders using the Rapid Office Strain Assessment (ROSA) Method and implementing ergonomics intervention programs in Sepah Bank. Health and Safety at Work. 2015 Jul 15;5(2):47-62.

45. Samaei SI, Tirgar A, Khanjani N, Mostafaee M, Bagheri Hosseinabadi M, Amrollahi M. Assessment of ergonomics risk factors influencing incidence of musculoskeletal disorders among office workers. Health and Safety at Work. 2015 Dec $15 ; 5(4): 1-2$. 